\title{
Super-replication of financial derivatives via convex programming
}

\author{
Nabil Kahalé *
}

November 12, 2012

\begin{abstract}
We give a method based on convex programming to calculate the optimal super-replicating and sub-replicating prices and corresponding hedging strategies of a financial derivative in terms of other financial derivatives. Our method finds a model that matches the superreplicating (or sub-replicating) price within an arbitrary precision and is consistent with the other financial derivatives prices. Applications include robust replication in terms of call prices with various strikes and maturities of forward start options, volatility and variance swaps and derivatives, cliquets calls, barrier options, lookback and Asian options. Numerical examples show that, in some cases, the super-replicating and/or sub-replicating prices are within $10 \%$ of the Black-and-Scholes price but considerably differ from it in other cases. Our method can take into account bid-ask spreads, interest rates and dividends and various limitations to the diffusion model. An alternative method to optimally super-replicate and sub-replicate forward start options using semi-definite and linear programming is presented.
\end{abstract}

Keywords: Model risk, robust replication, robust hedging, convex programming, financial derivatives.

\section{Introduction}

The valuation of financial derivatives is a question of fundamental importance. Non-liquid derivatives are often priced using models that are calibrated to fit the market prices of liquid financial products, such as vanilla options. Models that agree on the prices of liquid financial derivatives may produce different prices for more complex products such as barrier options (Britten-Jones and A. Neuberger 2000). Other financial derivatives such as variance swaps can be exactly replicated (Dupire 1993, Neuberger 1994) by a continuum set of call options if the underlying follows a continuous process. A July 2009 directive (Committee on Banking Supervision 2009) from the Basel Committee stipulates that "banks must explicitly assess the need for valuation adjustments to reflect two forms of model risk: the model risk associated with using a possibly incorrect valuation methodology; and the risk associated with using unobservable (and possibly incorrect) calibration parameters in the valuation model". This gives rise to the following questions:

1. What are the best model-free bounds on the price of a financial derivative based on a given set of financial derivatives prices?

2. Can we solve the above question if the size of the jumps the assets can take are restricted?

3. Can we solve the above questions by taking into account bid and ask prices?

These questions have been studied in the literature in special cases, either analytically or via optimization algorithms. For instance, a closed-form tight bound on the price of a call in terms

*ESCP Europe, 79 avenue de la République, 75543 Paris Cedex France; e-mail: nkahale@escpeurope.eu. 
of the forward price and of another moment of the underlying stock (Lo 1987, Grundy 1991) has been shown. Model-independent bounds on lookback and barrier options have been obtained by (Hobson 1998) and (Brown, Hobson and Rogers 2001) in terms of call prices with the same maturity and shown to be tight when interest rates are null. Semi-definite programming has been used to calculate an upper-bound (Boyle and Lin 1997) on a call on the maximum of several assets in terms of their means and correlations. Semi-definite tight bounds (Bertsimas and Popescu 2002, Gotoh and Konno 2002) on the price of an option on a single asset given several moments of the underlying asset have been established. A model-independent lower bound for prices of arithmetic Asian options (Albrecher, Mayer and Schoutens 2008) has been derived in terms of European call options. A tight but non-constructive upper bound (Hobson and Neuberger 2010) on the price of a forward start option on the forward price of the asset has been obtained. A tight lower bound for the price of a variance swap has been derived (Kahale 2009) in terms of call prices with the same maturity. Upper bounds and extensions of this result are given in (Hobson and Klimmek 2012).

This paper gives a unified methodology based on convex programming to calculate the best super-replicating and sub-replicating prices and corresponding hedging strategies of a financial derivative $\psi$ in terms of prices of a finite set of liquid derivatives in a multi-period setting. Further, it finds a model that matches the prices of the liquid financial derivatives and gives a price of $\psi$ arbitrarily close to the super-replicating (or sub-replicating) price, thereby proving the optimality of the latter. Applications include the calculation the best super-replicating and sub-replicating prices of a wide variety of derivatives such as forward start options, generalized variance swaps including cliquet calls and corridor variance swaps, volatility swaps and derivatives, lookback options, Asian options, single and double barrier options, in terms of call options with different strikes and maturities. We note that convex programming has already been used to super-replicate options (Bertsimas and Popescu 2002) in a single-period setting.

Definitions and preliminary results are given in section 2. Section 3 presents our convex program and shows that, under suitable conditions, it can be solved in polynomial time. Several examples including numerical applications are discussed in section 4. Section 5 shows our method can take into account bid-ask spreads, interest rates and dividends as well as various limitations to the diffusion model of the underlying securities, and discusses the single-maturity case. Section 6 gives an alternative method to calculate optimal super-replicating (resp. subreplicating) prices for forward-start options using semi-definite (resp. linear) programming. Section 7 contains concluding remarks.

\section{Preliminaries}

\subsection{The modelling framework}

Our modelling framework is inspired from the classical theory of multi-period markets (Pliska 2005, Chapter 3). We assume for simplicity and without loss of generality that interest rates are null, and consider $d$ basic securities whose prices $S_{0}^{1}, \ldots, S_{0}^{d}$ at time-step 0 are known constants. The price $S_{i}^{k}$ of security $k$ at time-step $i, 0 \leq i \leq m$, is a real-valued random variable on a non-empty sample space $\Omega$. Let $X_{i}$ be the price vector $\left(S_{i}^{1}, \ldots, S_{i}^{d}\right)$. An investor can buy $\xi_{i}^{k}$ positions in security $k, 1 \leq k \leq d$, at time-step $i-1$ and sell them at time-step $i$. Denote by $\xi_{i}$ the $d$-dimensional vector $\left(\xi_{i}^{k}\right), 1 \leq k \leq d$. The vector $\xi_{i}$ is an arbitrary function of the past values of the $d$ securities, i.e. it is a function of $S_{j}^{k}, 1 \leq k \leq d$, and $1 \leq j \leq i-1$. The cumulative payoff of the investor, which we call a gains function, is $\sum_{i=1}^{m} \xi_{i}^{T}\left(X_{i}-X_{i-1}\right)$. A finite-support probability is a non-negative function on $\Omega$ that takes positive values on a finite number of elements and that sums up to 1.

Definition 2.1. A risk-neutral probability is a finite-support probability $P$ such that $E_{P}(g)=0$ for any gains function $g$. 
An arbitrage opportunity is a non-negative gains function which is strictly positive for some $\omega \in \Omega$. Throughout the paper, we assume the market is arbitrage-free, which implies (Kahale 2010) the existence of a risk-neutral probability. A financial derivative is a real-valued random variable on $\Omega$. Dominating trading strategies is a notion related to arbitrage.

Definition 2.2. Let $\phi=\left(\phi_{k}\right), 1 \leq k \leq l$, be a vector of financial derivatives and $\pi=\left(\pi_{k}\right)$, $1 \leq k \leq l$, a price vector. A dominating trading strategy for $(\phi, \pi)$ consists of a vector $\beta \in \mathbb{R}^{l}$ and of a gains function $g$ such that

$$
\inf _{\omega \in \Omega} \beta^{T}(\phi(\omega)-\pi)+g(\omega)>0 .
$$

\subsection{Convex programming}

Let $C$ be a convex subset of $\mathbb{R}^{n}$. We say $C$ is well centered if it contains a known ball $B\left(a_{0}, r\right)$, where $a_{0} \in \mathbb{Q}^{n}, r>0$, and $B\left(a_{0}, r\right)$ is the ball centered at $a_{0}$ with radius $r$. By definition, $C$ is well bounded if it is contained in a known ball. We say an algorithm calculates a certain number $x$ (e.g. the root of an equation) in polynomial time if, for any $\epsilon>0$, the algorithm outputs a rational number within distance $\epsilon$ from $x$ and runs in time polynomial in the input size and in $\ln (1 / \epsilon)$.

Definition 2.3. A subset $C$ of $\mathbb{R}^{n}$ is admissible if there is a subset $D$ of $\mathbb{R}^{n} \times \mathbb{R}$ such that

$$
C=\left\{x \in \mathbb{R}^{n}: a^{T} x \geq b \text { for }(a, b) \in D\right\},
$$

with $\|a\| \geq 1$ for any $(a, b) \in D$, and there is a polynomial-time algorithm that, given a vector $y \in \mathbb{Q}^{n}$ and a rational number $\epsilon>0$, either

1. asserts that $y$ belongs to $C$ or

2. calculates a vector $(a, b) \in D$ such that $a^{T} y \leq b+\epsilon$ (i.e. one of the constraints 2.1 is almost violated).

In other words, a convex set $C$ given by Eq. 2.1 is admissible if, for any vector $y$, we can either assert in polynomial time that $y$ belongs to $C$ or give a proof that $y$ almost does not belong to $C$.

Example 2.1. A rational polytope is a subset $C$ of $\mathbb{R}^{n}$ defined by Eq. 2.1, where $D$ is a finite subset of $\mathbb{Q}^{n} \times \mathbb{Q}$. Any rational polytope is admissible.

The following version of the ellipsoid method shows that, under general conditions, linear optimization can be solved in polynomial time over an admissible set.

Theorem 2.1 (The ellipsoid method (Grötschel, Lovász and Schrijver 1981)). Consider a well centered, well bounded admissible subset $C$ of $\mathbb{R}^{n}$. Then there is a polynomial-time algorithm that, given a vector $a_{0} \in \mathbb{Q}^{n}$ and a rational number $\epsilon>0$, finds a vector $y \in C$ such that $a_{0}^{T} y \leq a_{0}^{T} x+\epsilon$ for every $x \in C$ (i.e. $y$ almost minimizes $a_{0}^{T} x$ over the points in $C$ ). Furthermore, the algorithm calculates a real number $b_{0}$ such that $\left(a_{0}, b_{0}\right)$ is an explicit linear combination of elements in $D$ with positive weights and $a_{0}^{T} y \leq b_{0}+\epsilon$.

Proof. See the appendix.

Thus the minimization algorithm gives a proof that $y$ almost minimizes $a_{0}^{T} x$ over $C$. Indeed, for any $x \in C, a_{0}^{T} x \geq b_{0}$ by Eq. 2.1, which implies that $a_{0}^{T} y \leq a_{0}^{T} x+\epsilon$.

The following is an immediate consequence of definition 2.3 .

Proposition 2.1. If $C$ and $C^{\prime}$ are admissible then $C \cap C^{\prime}$ is admissible. 


\section{Calculation of the super-replicating price}

We first cast the calculation of the super-replicating price in multi-period markets as a convex program. We then show how to solve this program using backward induction.

\subsection{Super-replication as a convex program}

Our method uses the following result.

Theorem 3.1 ((Kahale 2010)). Define the super-hedging price of financial derivative $\psi$

$$
c(\psi)=\inf _{g} \sup _{\omega \in \Omega} \psi(\omega)+g(\omega),
$$

where $g$ ranges over all gains functions. Then

$$
c(\psi)=\sup _{P \in \mathbb{P}} E_{P}(\psi),
$$

where $\mathbb{P}$ is the set of risk-neutral probabilities.

Let $\phi=\left(\phi_{1}, \ldots, \phi_{l}\right)$ be a vector of financial derivatives that trade at prices $\pi_{1}, \ldots, \pi_{l}$ at time-step 0. Consider a portfolio long in $\beta_{j}$ derivatives $\phi_{j}, 1 \leq j \leq l$, and in $\gamma$ bonds that pay 1 at time-step $m$. The cumulative payoff at time-step $m$ of the portfolio combined with a dynamic position in the basic securities is $g(\omega)+\beta^{T} \phi(\omega)+\gamma$, where $\beta=\left(\beta_{1}, \ldots, \beta_{l}\right)$ and $g$ is a gains function. The portfolio strictly super-replicates a financial derivative $\psi$ if and only if there is a gains function $g$ such that, for $\omega \in \Omega$,

$$
\psi(\omega)<g(\omega)+\beta^{T} \phi(\omega)+\gamma .
$$

By theorem 3.1, this is equivalent to $c\left(\psi-\beta^{T} \phi\right)<\gamma$. The super-replicating cost is $\beta^{T} \pi+\gamma$, where $\pi=\left(\pi_{1}, \ldots, \pi_{l}\right)$. Define the super-replicating price

$$
\pi_{\text {sup }}=\inf _{(\beta, \gamma) \in V} \beta^{T} \pi+\gamma
$$

where

$$
V=\left\{(\beta, \gamma) \in \mathbb{R}^{l} \times \mathbb{R}: c\left(\psi-\beta^{T} \phi\right) \leq \gamma\right\}
$$

Equivalently,

$$
\pi_{\text {sup }}=\inf _{\beta \in \mathbb{R}^{l}} c\left(\psi-\beta^{T}(\phi-\pi)\right)
$$

Note that, by theorem 3.1,

$$
V=\left\{(\beta, \gamma) \in \mathbb{R}^{l} \times \mathbb{R}: E_{P}(\psi) \leq \beta^{T} E_{P}(\phi)+\gamma \text { for } P \in \mathbb{P}\right\} .
$$

Hence $V$ is equal to the right-hand side of Eq. 2.1, with

$$
D=\left\{\left(\left(E_{P}(\phi), 1\right), E_{P}(\psi)\right): P \in \mathbb{P}\right\} .
$$

Thus $V$ is admissible if there is a polynomial-time algorithm that, on inputs $(\beta, \gamma)$ and $\epsilon>0$, either asserts that $(\beta, \gamma) \in V$ or calculates $E_{P}(\phi)$ and $E_{P}(\psi)$, where $P \in \mathbb{P}$ is such that

$$
\beta^{T} E_{P}(\phi)+\gamma \leq E_{P}(\psi)+\epsilon .
$$

More intuitively, given a portfolio of the derivatives $\phi_{j}$ and of bonds, we can assert in polynomial time that the portfolio, combined with appropriate dynamic hedging, super-replicates $\psi$ or give a proof that it almost does not.

Let $e_{i}$ be the vector of length $l$ whose $i$-th coordinate equals 1 and remaining coordinates are null. 
Theorem 3.2. Assume the following:

1. The convex set $V$ is admissible and contains a known ball $B(v, r)$.

2. There is $\delta>0$ and a rational $q$ such that, for

$$
\pi^{\prime} \in\left\{\pi \pm \delta e_{i}, 1 \leq i \leq l\right\}
$$

there is a risk-neutral probability $P$ with $E_{P}(\phi)=\pi^{\prime}$ and $q \leq E_{P}(\psi)$.

Let $a_{0}=(\pi, 1)$. Then

$$
\pi_{\text {sup }}=\inf _{(\beta, \gamma) \in V^{\prime}} \beta^{T} \pi+\gamma
$$

where

$$
V^{\prime}=\left\{(\beta, \gamma) \in V: \beta^{T} \pi+\gamma \leq a_{0}^{T} v+1\right\}
$$

is admissible, well bounded and well centered.

Proof. See the appendix.

Corollary 3.1. Under the assumptions of theorem 3.2, for any $\epsilon>0$, we can calculate in polynomial time a super-replicating portfolio $\left(\beta^{*}, \gamma^{*}\right) \in V^{\prime}$ and $E_{P}(\psi)$, where $P$ is a risk-neutral probability such that $E_{P}(\phi)=\pi$ and

$$
\pi_{\text {sup }} \leq \beta^{* T} \pi+\gamma^{*} \leq E_{P}(\psi)+\epsilon .
$$

Thus the algorithm calculates $\pi_{\text {sup }}$ with precision $\epsilon$ and outputs an $\epsilon$-optimal super-replicating portfolio together with the proof that it is e-optimal.

Proof. By theorem 2.1, we can calculate in polynomial time $\left(\beta^{*}, \gamma^{*}\right) \in V^{\prime}$ and an explicit linear combination

$$
\sum_{i=1}^{l} \lambda_{i}\left(\left(E_{P_{i}}(\phi), 1\right), E_{P_{i}}(\psi)\right)
$$

of elements of $D$ equal to $\left(a_{0}, b_{0}\right)$, with $\beta^{* T} \pi+\gamma^{*} \leq b_{0}+\epsilon$. Thus the probability $P=\sum_{i=1}^{l} \lambda_{i} P_{i}$ is risk-neutral and $E_{P}(\phi)=\pi$. Since $b_{0}=E_{P}(\psi)$, we conclude that

$$
\beta^{* T} \pi+\gamma^{*} \leq E_{P}(\psi)+\epsilon .
$$

Since $E_{P}(\psi)$ is upper-bounded by $\pi_{\text {sup }}$, the super-replicating cost in the left-hand side of Eq. 3.3, which is obviously lower-bounded by $\pi_{\text {sup }}$, is within $\epsilon$ from $\pi_{\text {sup }}$.

Remark 3.1. If, on inputs $\beta$ and $\epsilon>0$, we can calculate in polynomial time $E_{P}(\phi)$ and $E_{P}(\psi)$, where $P$ is a probability such that $c\left(\psi-\beta^{T} \phi\right) \leq E_{P}\left(\psi-\beta^{T} \phi\right)+\epsilon$, then $V$ is admissible.

Remark 3.2. If $c(-\psi)$ is upper-bounded by a known rational and $\left(\phi, \pi^{\prime}\right)$ does not give rise to a dominating trading strategy whenever Eq. 3.1 holds, then condition 2 of theorem 3.2 holds. This is because, by (Kahale 2010, theorem 4.6), there is a risk-neutral probability $P$ such that $E_{P}(\phi)=\left(\pi+\pi^{\prime}\right) / 2$. Furthermore, $-c(-\psi) \leq E_{P}(\psi)$ by theorem 3.1.

Remark 3.3. An optimal sub-replicating price and strategy for $\psi$ can be obtained by negating an optimal super-replicating price and strategy for $-\psi$. 


\subsection{Recursive calculation of the super-hedging cost}

We show how to calculate the super-hedging cost of a financial derivative by backward induction using concave envelopes, in the same spirit as in (Carassus, Gobet and Temam 2007).

Let $g$ be a real-valued function defined on a subset $W$ of $\mathbb{R}^{d}$ and bounded above by a linear function. Denote by $\hat{W}$ be the convex set spanned by $W$ and by $\bar{g}$ the concave envelope of $g$, i.e. the smallest concave function on $\hat{W}$ bounded below by $g$ on $W$. Using the separating hyperplane theorem, it can be shown that for $x \in \hat{W}$,

$$
\begin{aligned}
\bar{g}(x) & =\sup E_{\mathcal{Q}}(g) \\
& =\inf _{\eta \in \mathbb{R}^{d}} \sup _{y \in W} g(y)+\eta^{T}(y-x),
\end{aligned}
$$

where $\mathcal{Q}$ ranges over finite-support probabilities on $W$ with expectation equal to $x$. Eq. 3.5 says that $g(x)$ is the infimum value of $G(x)$, where $G$ ranges over the linear functions that upper-bound $g$ on $W$. Consider a financial derivative $\psi$ of the form $\psi=\psi^{*}\left(X_{1}, \ldots, X_{m}\right)$, where $\psi^{*}$ is a real-valued function defined on $\operatorname{Im}\left(X_{1}, \ldots, X_{m}\right)$. Assume $c(\psi)$ is finite. When $m=1$, it follows from Eq. 3.5 that

$$
c(\psi)=\overline{\psi^{*}}\left(X_{0}\right) .
$$

We now show how to calculate the super-hedging cost in a multi-period market for any integer $m$. Denote by $\psi_{i}, 0 \leq i \leq m-1$, the super-hedging cost at time-step $i$ of $\psi$. We will show that $\psi_{i}$ is equal to the super-hedging cost of $\psi_{i+1}$ in the underlying single-period market at time-step $i$. Thus $\psi_{i}$ is almost equal to the expected value of $\psi_{i+1}$ under a risk neutral probability in the single-period market at time-step $i$. As in (Pliska 2005, Section 3.4), we can paste together these probabilities by multiplying them along any given path to obtain a risk-neutral probability in the $m$-period market. We show below that the expected value of $\psi$ under the resulting probability is almost equal to $c(\psi)$.

More formally, for $0 \leq i \leq m$, let $D_{i}=\operatorname{Im}\left(X_{1}, \ldots, X_{i}\right)$ be the set of paths $X$ can follow from steps 1 through $i$. By convention, $D_{0}=\{\emptyset\}$. For $\theta \in D_{i}$, let

$$
D(\theta)=\left\{x \in \mathbb{R}^{d}:(\theta, x) \in D_{i+1}\right\}
$$

be the set of possible values of $X_{i+1}$ given that $X$ has followed the path $\theta$ in the first $i$ steps. It might be easier for the reader to think of $D(\theta)$ as a constant set, e.g. $\mathbb{R}^{+}$, which will be the case in many applications. If $\zeta$ is a real-valued function over $D_{i+1}$, denote by $\zeta(\theta,$.$) the function$ that maps $x$ to $\zeta(\theta, x)$ for $x \in D(\theta)$. Fix $\epsilon>0$.

Proposition 3.1. Define the functions $\psi_{i}^{*}$ by backward induction as follows: $\psi_{m}^{*}=\psi^{*}$ and $\psi_{i}^{*}(\theta)=\overline{\psi_{i+1}^{*}(\theta, .)}\left(x_{i}\right)$ for $0 \leq i \leq m-1$ and $\theta=\left(x_{1}, \ldots, x_{i}\right) \in D_{i}$, where $x_{0}=X_{0}$ by convention. Then $c(\psi)=\psi_{0}$ and

$$
\psi_{i}^{*}(\theta)=\sup _{\mathcal{Q} \in \mathbb{P}(\theta)} E_{\mathcal{Q}}\left(\psi_{i+1}^{*}(\theta, .)\right)
$$

where $\mathbb{P}(\theta)$ is the set of finite-support probabilities defined on $D(\theta)$ with expected value equal to $x_{i}$. There is a random variable $\xi_{i+1}$ which is a function of $X_{0}, \ldots, X_{i}$ such that

$$
\psi_{i+1} \leq \epsilon+\xi_{i+1}^{T}\left(X_{i+1}-X_{i}\right)+\psi_{i},
$$

where $\psi_{i}$ is the financial derivative $\psi_{i}^{*}\left(X_{1}, \ldots, X_{i}\right)$.

Proof. See the appendix.

Remark 3.4. A portfolio long in $\psi_{0}$ bonds that pay 1 at time-step $m$ combined with a dynamic strategy that buys $\xi_{i+1}$ basic securities at time-step $i$ and sells them at time-step $i+1$ super-hedges $\psi$ up to the additive constant $m \epsilon$. 
Let $\phi$ be a financial derivative of the form $\phi=\bar{\phi}\left(X_{1}, \ldots, X_{m}\right)$.

Proposition 3.2. For $\theta \in D_{i}$, choose $\mathcal{P}_{\theta} \in \mathbb{P}(\theta)$ that attains the right-hand side of $E q$ 3.7 within precision $\epsilon$. Define the functions $\bar{\phi}_{i}$ on $D_{i}$ by backward induction as follows: $\bar{\phi}_{m}=\bar{\phi}$ and

$$
\bar{\phi}_{i}(\theta)=E_{\mathcal{P}_{\theta}}\left(\bar{\phi}_{i+1}(\theta, .)\right)
$$

for $0 \leq i \leq m-1$ and $\theta \in D_{i}$. There is a risk-neutral probability $P$ that does not depend on $\phi$ such that $c(\psi) \leq E_{P}(\psi)+m \epsilon$ and $E_{P}(\phi)=\bar{\phi}_{0}(\emptyset)$.

Proof. See the appendix.

Remark 3.5. It can be shown by backward induction that if $\psi=\eta+\nu$, where the random variable $\eta($ resp. $\nu)$ is a deterministic function of $X_{0}, \ldots, X_{m}\left(\right.$ resp. $\left.X_{0}, \ldots, X_{i}\right)$, then $\psi_{i}=\eta_{i}+\nu$.

Remark 3.6. Fix $i \in\{1, \ldots, m-1\}$. Assume that $\psi^{*}$ and $D\left(x_{1}, \ldots, x_{j}\right)$ depend on $\left(x_{1}, \ldots, x_{i}\right)$ only through a (one or multi-dimensional) function $h\left(x_{1}, \ldots, x_{i}\right)$ for $j \geq i$. It can be shown by backward induction that $\psi_{i}^{*}$ is a function of $x_{i}$ and of $h\left(x_{1}, \ldots, x_{i}\right)$.

\subsection{Practical implementation issues}

When $m=1$, it follows from Eq. 3.6 that $\left(X_{0}, c(\psi)\right)$ belongs to the upper hull of the set $F=\left\{\left(x, \psi^{*}(x)\right): x \in \operatorname{Im}\left(X_{1}\right)\right\}$, i.e. the portion of the convex hull which is visible from above. If $d=1$ and $F$ is finite and given in increasing order along the first coordinate, then $c(\psi)$ can be calculated in time proportional to the size of $F$. This is because the upper hull of a sorted set of planar points (Andrew 1979) can be calculated in linear time, as described in proposition 3.3. Let $x^{\prime}$ and $x^{\prime \prime}$ be distinct elements of $\operatorname{Im}\left(X_{1}\right)$ such that $\left(X_{0}, c(\psi)\right)$ belongs to the segment joining $\left(x^{\prime}, \psi^{*}\left(x^{\prime}\right)\right)$ to $\left(x^{\prime \prime}, \psi^{*}\left(x^{\prime \prime}\right)\right)$. Let $\xi_{1}$ be the slope of this segment and let $q=\left(x^{\prime \prime}-X_{0}\right) /\left(x^{\prime \prime}-x^{\prime}\right)$. The probability $\mathcal{Q}$ that assigns $q$ to $x^{\prime}$ and $1-q$ to $x^{\prime \prime}$ has mean $X_{0}$ and $E_{\mathcal{Q}}\left(\psi^{*}\right)=c(\psi)$. Furthermore, Eq. 3.8 holds with $\epsilon=0$ and $i=0$.

Proposition 3.3 ((Andrew 1979)). Let $\left(p_{1}, \ldots, p_{n}\right)$ be a sequence of $n$ points in the plane sorted from left to right. Let $U C H(j)$ be the upper convex hull of the first $j$ points. Then $\operatorname{UCH}(2)=\left(p_{1}, p_{2}\right)$. Assume $\operatorname{UCH}(j-1)=\left(p_{1}^{\prime}, \ldots, p_{h}^{\prime}\right)$. Let $k$ be the largest index such that $p_{k}^{\prime}$ is above the segment $\left[p_{k-1}^{\prime}, p_{j}\right]$, if such an index exists, otherwise let $k=1$. Then $U C H(j)=\left(p_{1}^{\prime}, \ldots, p_{k}^{\prime}, p_{j}\right)$.

For $m>1$, proposition 3.1 implies that $\left(x_{i}, \psi_{i}^{*}(\theta)\right)$ belongs to the upper hull of the set $F=\left\{\left(x, \psi_{i+1}^{*}(\theta, x)\right): x \in D(\theta)\right\}$. As in the case $m=1$, if $d=1$ and $F$ is finite and given in increasing order along the first coordinate, then $\psi_{i}^{*}(\theta)$, a probability $\mathcal{Q}$ that maximises the right-hand side of Eq 3.7 and a vector $\xi_{i+1}$ such that Eq. 3.8 holds with $\epsilon=0$ can be calculated in time proportional to the size of $F$.

In our numerical implementation, we have solved the convex program 3.2 using the analytic center cutting plane algorithm. Calculation of the analytic center also gives a direct proof of the optimality of the solution to the convex program, without the need to perform linear programming. In practice, the knowledge of $q$ and of $\delta$ in theorem 3.1 is not necessary. It is sufficient to guess boundaries to the set $V^{\prime}$. If the solution to the convex program 3.2 using these boundaries finds a risk-neutral probability $P$ such that $E_{P}(\phi)$ almost equals $\pi$ and $E_{P}(\psi)$ almost matches the super-replicating price, our initial guess is correct. Otherwise, we enlarge our boundaries and repeat the same process again. 


\section{Examples}

Consider a stock $S$ valued at $S_{i}$ at time-step at time-step $i, 0 \leq i \leq m$, where $S_{0}$ is known. Assume that $S_{i}$ ranges in a subset $D$ of $\mathbb{R}^{+}$for $1 \leq i \leq m$. For $1 \leq i \leq m$, we are given an increasing sequence $K_{i, j}$ of positive strikes, $1 \leq j \leq l_{i}$, together with prices $c_{i, j}$ of calls with maturity $t_{i}$ and strike $K_{i, j}$ that strictly satisfy the usual no-arbitrage conditions (see (Davis and Hobson 2007)). We assume that $D$ is sufficiently dense and has a sufficiently large range so that the call prices, even under a small perturbation and the restriction that $S_{i} \in D$, induce no dominating trading strategies. For $x \geq 0$ and $1 \leq i \leq m$, define the vector $f_{i}(x)=$ $\left(\max \left(x-K_{i, j}, 0\right)\right), 1 \leq j \leq l_{i}$, and let $b_{i} \in \mathbb{R}^{l_{i}}$. Consider the vector of calls $\phi=\left(f_{i}\left(S_{i}\right)\right)$ and set $\beta=\left(b_{i}\right), 1 \leq i \leq m$. Let $\psi$ be a financial derivative that pays $\psi^{*}\left(S_{1}, \ldots, S_{m}\right)$, where $\psi^{*}$ is a deterministic function. Assume that $c(\psi)$ and $c(-\psi)$ are upper bounded by a known rational number. We use corollary 3.1 and remark 3.4 in the following examples to calculate an optimal super-replicating price and strategy for $\psi$ via the call components of $\phi$. An optimal sub-replicating price and strategy can be derived as well using remark 3.3. Consider the financial derivative $\Psi=\psi-\beta^{T} \phi$. Since $c$ is sub-additive, $c(\Psi) \leq c(\psi)+\|\beta\|_{1} S_{0}$, the set $V$ contains a known ball. Furthermore, by remark 3.2, condition 2 of theorem 3.2 holds. In the following examples, we show that $V$ is admissible using remark 3.1. Our numerical examples were obtained using an analytic center cutting plane algorithm with at most 150 iterations that yielded a super-replicating (resp. sub-replicating) price and a risk-neutral probability that simultaneously matched the super-replicating (resp. sub-replicating) price and the call prices within an error of order $10^{-5}$ or less in terms of the notional. The notional is $S_{0}$ for call and forward start options and 1 for variance swaps and volatilities.

\subsection{Forward start options}

Consider a forward start option $\psi$. For ease of exposition, assume the option is an at the money call that pays $\max \left(0, S_{2}-S_{1}\right)$ at maturity. Extension to the general case is straightforward. We show that $V$ is admissible when $D$ is finite. The derivative $\Psi$ pays $\Psi^{*}\left(S_{1}, S_{2}\right)$, where

$$
\Psi^{*}\left(x_{1}, x_{2}\right)=\max \left(0, x_{2}-x_{1}\right)-b_{1}^{T} f_{1}\left(x_{1}\right)-b_{2}^{T} f_{2}\left(x_{2}\right) .
$$

By proposition 3.1, for $x_{1} \in D$,

$$
\Psi_{1}^{*}\left(x_{1}\right)=\sup _{\mathcal{Q}} E_{\mathcal{Q}}\left(\Psi^{*}\left(x_{1}, .\right)\right)
$$

where $\mathcal{Q}$ ranges over all probabilities on $D$ whose expected value equals $x_{1}$, and

$$
c(\Psi)=\sup _{\mathcal{Q}^{\prime}} E_{\mathcal{Q}^{\prime}}\left(\Psi_{1}^{*}\right),
$$

where $\mathcal{Q}^{\prime}$ ranges over all probabilities on $D$ whose expected value equals $S_{0}$. Subsection 3.3 shows how to calculate $\Psi_{1}^{*}\left(x_{1}\right)$ for all $x_{1} \in D$ and probabilities that maximise the right-hand side of Eqs 4.1 and 4.2 in total running time proportional to $|D|^{2}$. Using Proposition 3.2, we can then calculate $E_{P}(\phi)$ and $E_{P}(\psi)$, where $P$ is a risk-neutral probability such that $c(\Psi)=E_{P}(\Psi)$. By remark 3.1, we conclude that $V$ is admissible.

We illustrate the calculation of $c(\Psi)$ with a simple example. Let $S_{0}=100$ and $D=$ $\{70,80, \ldots, 130\}$. Assume $\phi$ consists of the calls maturing at time-step $i$ with strike $K_{i, j}=$ $80+10 j$ for $1 \leq i \leq 2$ and $1 \leq j \leq 3$. Let $b_{1}=(-0.3,-0.2,-0.4)$ and $b_{2}=(0.5,0.4,0.3)$. Table 1 lists the values of $\Psi$ in the case where $S_{1}=90$. Fig. 1 plots the function $\Psi^{*}(90,$.$) and$ its concave envelope, which shows that $\Psi_{1}^{*}(90)=2 / 3 \times 5+1 / 3 \times 0=10 / 3$. The probability that assigns a weight $2 / 3$ to 100 and $1 / 3$ to 70 maximises the right-hand side of Eq 4.1 when $x_{1}=90$. We can perform a similar calculation for each $x_{1} \in D$ and then calculate $c(\Psi)$ via Eq. 4.2. Fig. 2 draws a tree by connecting each $x_{1} \in D$ (resp. $S_{0}$ ) to the support of a probability that maximises 


\begin{tabular}{|c|c|c|c|c|c|c|c|}
\hline$S_{2}$ & 70 & 80 & 90 & 100 & 110 & 120 & 130 \\
\hline$\Psi^{*}(90,)$. & 0 & 0 & 0 & 5 & 6 & 4 & 2 \\
\hline
\end{tabular}

Table 1: The function $\Psi^{*}(90,$.$) .$

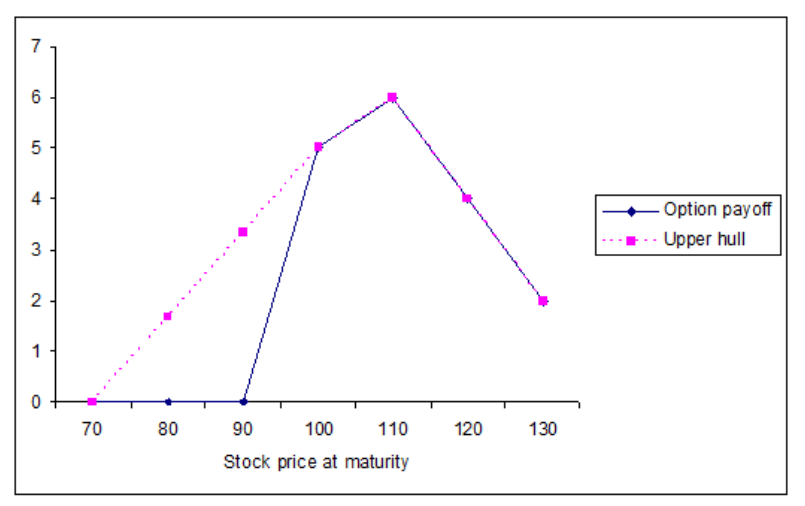

Figure 1: The solid line plots the payoff of $\Psi$ when $S_{1}=90$ and the dotted line plots the corresponding upper hull.

the right-hand side of Eq 4.1 (resp. Eq. 4.2). Table 2 gives the probability $p_{u}$ assigned to the largest element in the support of such a probability and shows that $E_{P}(\eta)=\$ 7.5$, where $\eta$ is the ATM vanilla call maturing at the second time-step and $P$ is a risk-neutral probability such that $c(\Psi)=E_{P}(\Psi)$. The expected value under $P$ of any component of $\phi$ can be calculated in a similar manner.

We now consider the case where $D$ is equal to the set

$$
\left\{S_{0} j / n\right\} \cup\left\{S_{0}\left(M / S_{0}\right)^{j / n}\right\}
$$

with $S_{0}=\$ 100, n=10^{4}, M=10^{5}$ and $j \in\{0, \ldots, n\}$. Let $t_{1}=1 / 6$ (two months) and $t_{2}=5 / 12$ (five months), where $t_{i}$ is the maturity of time-step $i$. Assume the market price $c_{i, j}$ of the call maturing at time-step $i$ with strike $K_{i, j}=60+10 j$ is equal to the Black-and-Scholes price with the corresponding strike, maturity $t_{i}$ and volatility $\sigma=0.2$ for $1 \leq i \leq 2$ and $1 \leq j \leq 7$. Table 3 gives the optimal super-replicating price and the calls coefficients in the corresponding super-replicating portfolio. The sub-replicating price was derived using remark 3.3.

Remark 4.1. Using Eqs. 4.1, 4.2 and the techniques of Section 6, it is possible to calculate $c(\Psi)$ via a closed-form formula when $D=\mathbb{R}^{+}$.

\begin{tabular}{|c|c|c|c|c|c|c|c|c|}
\hline node & A & B & C & D & E & F & G & H \\
\hline$p_{u}$ & $1 / 2$ & 1 & $2 / 3$ & $1 / 2$ & $1 / 2$ & $2 / 3$ & $1 / 2$ & 1 \\
\hline$\Psi_{i}$ & 1.1666 & -12 & -3.3333 & -1 & 1 & 3.3333 & 5 & 0 \\
\hline $\bar{\eta}_{i}$ & 7.5 & 30 & 20 & 15 & 5 & 0 & 0 & 0 \\
\hline
\end{tabular}

Table 2: The probabilities $p_{u}$ of an "up" movement and the values of $\Psi_{i}$ (resp. $\bar{\eta}_{i}$ ) calculated via proposition 3.1 (resp. proposition 3.2) by backward induction inside the tree of Fig. 2, where $i$ is the time-step of the corresponding node. 


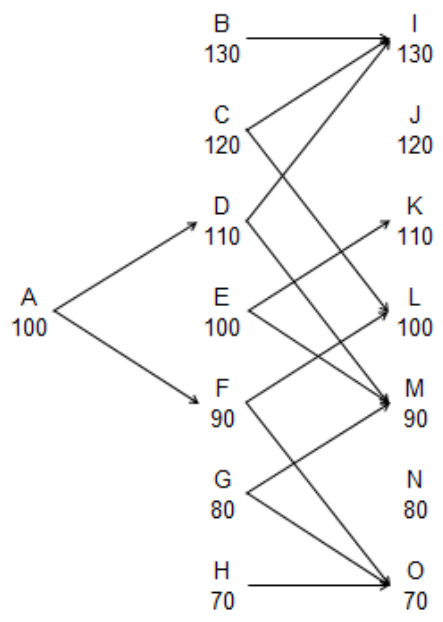

Figure 2: Connecting each $x_{1} \in D$ (resp. $S_{0}$ ) to the support of a probability that maximises the right-hand side of Eq 4.1 (resp. Eq. 4.2).

\begin{tabular}{|c|c|c|c|c|c|c|c|}
\hline Strike & 70 & 80 & 90 & 100 & 110 & 120 & 130 \\
\hline$b_{1}$ & 0.3657 & -0.2753 & -0.4690 & -0.4298 & -0.4677 & 0.3080 & 0.0015 \\
\hline$b_{2}$ & 0.4729 & 0.4602 & 0.4388 & 0.4602 & 0.4383 & 0.4590 & 0.2469 \\
\hline
\end{tabular}

Table 3: Optimal super-replication of the forward-start option via calls. The optimal superreplicating price is $\$ 5.2750$. The optimal sub-replicating price is $\$ 1.9363$. The Black-andScholes price is $\$ 3.9878$. When $D=\mathbb{R}^{+}$, the best super-replicating price calculated without discretization using the techniques of Section 6 is $\$ 5.2755$.

\subsection{Variance swaps}

Consider a variance swap that pays at maturity $T$ the amount

$$
\psi=\sum_{i=1}^{m} H\left(S_{i-1}, S_{i}\right)
$$

where $H$ is a deterministic bivariate function. For instance, $H(x, y)=T^{-1} \ln ^{2}(y / x)$ for standard variance swaps, $H(x, y)=T^{-1} \ln ^{2}(y / x) 1_{y \in I}$ for a corridor variance swaps, where $I$ is a specified interval of $\mathbb{R}^{+}$, and $H(x, y)=\max (0, y / x-K)$ for a cliquet call, where $K$ is a constant. In practice, $m$ is quite large and $l_{i}=0$ for most values of $i$. Let $H_{i}(x, y)=H(x, y)-b_{i}^{T} f_{i}(y)$, so that $\Psi=\sum_{i=1}^{m} H_{i}\left(S_{i-1}, S_{i}\right)$. For a given $i$, consider the financial derivative $\eta=\sum_{j=i+1}^{m} H_{j}\left(S_{j-1}, S_{j}\right)$. Since $\eta$ does not depend on $S_{0}, \ldots, S_{i-1}$, Remark 3.6 shows that the super-hedging cost of $\eta$ at time-step $i$ depends only on $S_{i}$. In other words, $\eta_{i}=c_{i}\left(S_{i}\right)$, where $c_{i}$ is a deterministic function on $D$. Since $\Psi=\eta+\sum_{j=1}^{i} H_{j}\left(S_{j-1}, S_{j}\right)$, remark 3.5 shows that

$$
\Psi_{i}=c_{i}\left(S_{i}\right)+\sum_{j=1}^{i} H_{j}\left(S_{j-1}, S_{j}\right)
$$

and so, by Eq. 3.7,

$$
c_{i}(x)=\sup _{\mathcal{Q}} E_{\mathcal{Q}}\left(c_{i+1}+H_{i+1}(x, .)\right)
$$

for $x \in D$, where $\mathcal{Q}$ ranges over all probabilities on $D$ whose expected value equals $x$.

As in the forward-start example, when $D$ is finite, the functions $c_{i}$ and probabilities $\mathcal{Q}$ maximizing the right-hand side of Eq. 4.4 can be calculated by backward induction in time proportional to $m|D|^{2}$. Proposition 3.2 then shows how to calculate $E_{P}(\phi)$ and $E_{P}(\psi)$, where 


\begin{tabular}{|c|c|c|c|c|c|c|c|}
\hline$\sigma$ & $10 \%$ & $15 \%$ & $20 \%$ & $25 \%$ & $30 \%$ & $35 \%$ & $40 \%$ \\
\hline$\sqrt{\pi_{\text {sup }}}$ & $12.43 \%$ & $16.92 \%$ & $21.76 \%$ & $26.81 \%$ & $32.01 \%$ & $37.38 \%$ & $42.94 \%$ \\
\hline$\sqrt{\pi_{\text {inf }}}$ & $8.68 \%$ & $13.90 \%$ & $18.91 \%$ & $23.77 \%$ & $28.52 \%$ & $33.19 \%$ & $37.79 \%$ \\
\hline
\end{tabular}

Table 4: Super-replicating and sub-replicating prices of variances swaps maturing in one month.

\begin{tabular}{|c|c|c|c|c|c|c|c|c|c|c|c|c|c|}
\hline Strike & 70 & 75 & 80 & 85 & 90 & 95 & 100 & 105 & 110 & 115 & 120 & 125 & 130 \\
\hline$b_{\text {sup }}$ & 0.095 & 0.108 & 0.030 & 0.023 & 0.018 & 0.014 & 0.012 & 0.012 & 0.011 & 0.011 & 0.010 & 0.010 & 0.119 \\
\hline$b_{\text {inf }}$ & -0.030 & -0.037 & 0.015 & 0.014 & 0.013 & 0.012 & 0.012 & 0.010 & 0.008 & 0.006 & 0.004 & 0.003 & -0.020 \\
\hline
\end{tabular}

Table 5: Optimal super-replicating $\left(b_{\text {sup }}\right)$ and sub-replicating $\left(b_{\text {inf }}\right)$ portfolios for a variance swap when $\sigma=0.2$.

$P$ is a risk-neutral probability such that $c(\Psi)=E_{P}(\Psi)$. For standard variance swaps, we assume that $D$ does not contains 0 so that $c(\psi)$ is finite.

In our numerical example, we assume that $S_{0}=\$ 100$ and that $D$ consists of $L(M / L)^{j / n}$, where $j \in\{0, \ldots, n\}$, with $L=\$ 50, M=\$ 200$ and $n=1000$. The variance swap has a maturity of one month with $m=20$ daily observations. Assume the market price $c_{m, j}$ of the call maturing at time-step $i$ with strike $K_{m, j}=65+5 j$ is equal to the Black-and-Scholes price with the corresponding strike, maturity one month and volatility $\sigma$ for $1 \leq j \leq 13$ and that no other call prices are known. Table 4 lists the optimal super-replicating and sub-replicating prices in terms of the volatility, and table 5 gives the calls coefficients in the corresponding portfolios when $\sigma=0.2$.

\subsection{Barrier options}

Consider an up-and-in barrier option $\psi$ that pays off $g\left(S_{T}\right)$ at maturity $T$ if $S_{i} \geq L$ for some $i \in\{1, \ldots, m\}$, and pays 0 otherwise, where $L>S_{0}$ is a constant and $g$ is the payoff of a vanilla option struck at $K$. Assume that $D=\mathbb{R}^{+}$. Given $i$, define the financial derivative

$$
\eta=\psi-\sum_{j=i+1}^{m} b_{j}^{T} f_{j}\left(S_{j}\right)
$$

Remark 3.6 shows that the super-hedging cost of $\eta$ at time-step $i$ depends only on $S_{i}$ and on whether the barrier has been hit before step $i$. In other words,

$$
\eta_{i}=c_{i}\left(t_{i}, S_{i}\right)
$$

where $c_{i}$ is a deterministic function and

$$
t_{i}=\left\{\begin{array}{l}
1 \text { if } S_{j}<L \text { for } j<i \\
0 \text { otherwise. }
\end{array}\right.
$$

Letting $i=m$ in Eq. 4.5 shows that $c_{m}(0, x)=g(x)$ and $c_{m}(1, x)=1_{L \leq x} g(x)$. Since $\Psi=$ $\eta-\sum_{j=1}^{i} b_{j}^{T} f_{j}\left(S_{j}\right)$, remark 3.5 shows that

$$
\Psi_{i}=c_{i}\left(t_{i}, S_{i}\right)-\sum_{j=1}^{i} b_{j}^{T} f_{j}\left(S_{j}\right) .
$$

Thus, by Eq. 3.7 and the observation that $t_{i+1}=t_{i} 1_{S_{i}<L}$,

$$
c_{i}(t, x)=\sup _{\mathcal{Q}} E_{\mathcal{Q}}\left(c_{i+1}\left(t 1_{x<L}, .\right)-b_{i+1}^{T} f_{i+1}\right),
$$

where $\mathcal{Q}$ ranges over all probabilities on $\mathbb{R}^{+}$whose expected value equals $x$. 


\begin{tabular}{|c|c|c|c|c|c|c|c|}
\hline$\sigma$ & $10 \%$ & $15 \%$ & $20 \%$ & $25 \%$ & $30 \%$ & $35 \%$ & $40 \%$ \\
\hline$\pi_{\text {sup }}$ & $12.26 \%$ & $16.62 \%$ & $21.27 \%$ & $26.06 \%$ & $30.94 \%$ & $35.88 \%$ & $40.92 \%$ \\
\hline$\pi_{\text {inf }}$ & $4.03 \%$ & $6.06 \%$ & $8.06 \%$ & $10.01 \%$ & $11.84 \%$ & $13.80 \%$ & $15.59 \%$ \\
\hline
\end{tabular}

Table 6: Super-replicating and sub-replicating prices of a volatility swap maturing in one month and capped at $\sigma \sqrt{2.5}$. In our implementation, the realized volatility $\sqrt{T^{-1} \nu}$ can take 50 equally spaced values.

\begin{tabular}{|c|c|c|c|c|c|c|c|c|c|c|c|c|c|}
\hline Strike & 70 & 75 & 80 & 85 & 90 & 95 & 100 & 105 & 110 & 115 & 120 & 125 & 130 \\
\hline$b_{\text {sup }}$ & 0.236 & 0.265 & 0.039 & 0.036 & 0.033 & 0.031 & 0.028 & 0.026 & 0.024 & 0.023 & 0.021 & 0.019 & 0.086 \\
\hline$b_{\text {inf }}$ & -0.141 & -0.156 & 0.000 & 0.000 & -0.026 & -0.009 & 0.042 & -0.008 & -0.025 & 0.000 & 0.000 & 0.000 & -0.002 \\
\hline
\end{tabular}

Table 7: Optimal super-replicating $\left(b_{\text {sup }}\right)$ and sub-replicating $\left(b_{\text {inf }}\right)$ portfolios for a capped volatility swap when $\sigma=0.2$.

Eq. 4.6 implies that $c_{i}(t,$.$) is an affine function on each interval not intersecting the set$ $\{K, L\} \cup\left\{K_{i^{\prime}, j}: i^{\prime}>i\right\}$ and can be calculated recursively by backward induction. Eq. 4.5 and proposition 3.1 show that $c(\Psi)=c_{0}\left(1, S_{0}\right)$. Furthermore, for any $\epsilon>0$, a probability that maximizes the right-hand side of Eq. 4.6 within precision $\epsilon$ can be found in polynomial time. Proposition 3.2 then shows how to calculate $E_{P}(\phi)$ and $E_{P}(\psi)$, where $P$ is a risk-neutral probability such that $c(\Psi) \leq E_{P}(\Psi)+\epsilon$.

\subsection{Volatility swaps}

Consider a volatility swap that pays at maturity $T$ the amount

$$
\psi=\sqrt{T^{-1} \sum_{i=1}^{m} \ln ^{2}\left(\frac{S_{i}}{S_{i-1}}\right)}
$$

In market practice, the payment is sometimes capped at a fixed amount. Assume $D=$ $\left\{L(M / L)^{j / n}: 0 \leq j \leq n\right\}$, where $L<S_{0}<M$ and integer $n$ are fixed. Given $i$, define the financial derivative

$$
\eta=\psi-\sum_{j=i+1}^{m} b_{j}^{T} f_{j}\left(S_{j}\right)
$$

Remark 3.6 shows that the super-hedging cost of $\eta$ at time-step $i$ depends only on $S_{i}$ and on the $\operatorname{sum} \nu_{i}=\Sigma_{j=1}^{i} \ln ^{2}\left(S_{j} / S_{j-1}\right)$. In other words, $\eta_{i}=c_{i}\left(\nu_{i}, S_{i}\right)$, where $c_{i}$ is a deterministic function. Eq. 4.7 shows that $c_{m}(\nu, x)=\sqrt{T^{-1} \nu}$. Since $\Psi=\eta-\sum_{j=1}^{i} b_{j}^{T} f_{j}\left(S_{j}\right)$, remark 3.5 shows that

$$
\Psi_{i}=c_{i}\left(\nu_{i}, S_{i}\right)-\sum_{j=1}^{i} b_{j}^{T} f_{j}\left(S_{j}\right) .
$$

Thus, by Eq. 3.7 and the observation that $\nu_{i+1}=\nu_{i}+\ln ^{2}\left(S_{i+1} / S_{i}\right)$,

$$
c_{i}(\nu, x)=\sup _{\mathcal{Q}} E_{\mathcal{Q}}\left(g-b_{i+1}^{T} f_{i+1}\right)
$$

where $\mathcal{Q}$ ranges over all probabilities on $D$ whose expected value equals $x$, and $g(z)=c_{i+1}(\nu+$ $\left.\ln ^{2}(z / x), z\right)$ for $z \in D$. As in the previous examples, the functions $c_{i}$ and probabilities $\mathcal{Q}$ maximizing the right-hand side of Eq. 4.8 can be calculated by backward induction when $\nu$ is a multiple of $n^{-2} \ln ^{2}(M / L)$ and $x \in D$. Table 6 lists the optimal super-replicating and subreplicating prices for a capped volatility swap using the same setting as in Table 4 except that $n=400$, and table 7 gives the calls coefficients in the corresponding portfolios when $\sigma=0.2$. 


\subsection{Other financial derivatives}

Other financial derivatives such as lookback options, options on realized variance and realized volatility, double barrier options and Asian options can be handled in a similar fashion. Consider for instance an Asian call $\psi$ that pays

$$
\max \left(\frac{S_{1}+\cdots S_{m}}{m}-K, 0\right)
$$

Assume $D=\{j M / n: 0 \leq j \leq n\}$, where $M>S_{0}$ and integer $n$ are fixed. Using the same techniques as before, we can show that

$$
\Psi_{i}=c_{i}\left(\nu_{i}, S_{i}\right)-\sum_{j=1}^{i} b_{j}^{T} f_{j}\left(S_{j}\right),
$$

where $\nu_{i}=\Sigma_{j=1}^{i-1} S_{j}$. We calculate the functions $c_{i}$ by backward induction by noting that $c_{m}(\nu, x)=\max ((\nu+x) / m-K, 0)$ and

$$
c_{i}(\nu, x)=\sup _{\mathcal{Q}} E_{\mathcal{Q}}\left(c_{i+1}(\nu+x, .)-b_{i+1}^{T} f_{i+1}\right),
$$

where $\mathcal{Q}$ ranges over all probabilities on $D$ whose expected value equals $x$.

We can also derive optimal replicating prices and strategies for the financial derivatives considered in this section in terms of prices of moments (or of the logarithmic function) of the stock price at different maturities rather than in terms of call prices.

\section{Extensions}

\subsection{Taking interest rates and dividends into account}

Interest rates can be taken into account in the usual way by replacing each security by its discounted value. We can also incorporate dividends in our model by replacing each security by the value of the security plus reinvested dividends, using a technique similar to the one in (Pliska 2005, SubSec 3.2.3).

\subsection{Taking bid-ask spreads into account}

Assume now the financial derivatives $\phi_{k}, 1 \leq k \leq l$, have distinct bid and ask prices, and let $\pi^{b}$ (resp. $\pi^{a}$ ) be the length $l$ vector of bid (resp. ask) prices. Results in the preceding sections can be easily extended to this case. For instance, theorem 3.2 can be replaced as follows.

Theorem 5.1. Assume the following:

1. The set $V$ is admissible and contains a known ball $B(v, r)$.

2. There is $\delta>0$, a rational $q$ and a risk-neutral probability $P$ such that $E_{P}(\phi) \in\left[\pi^{b}+\right.$ $\left.\delta \mathbf{1}, \pi^{\mathbf{a}}-\delta \mathbf{1}\right]$ and $q \leq E_{P}(\psi)$.

Let $a_{0}=\left(\pi^{a},-\pi^{b}, 1\right)$. Then

$$
\pi_{\text {sup }}=\inf _{\left(\beta^{a}, \beta^{b}, \gamma\right) \in V^{\prime}} a_{0}^{T}\left(\beta^{a}, \beta^{b}, \gamma\right)
$$

where

$$
V^{\prime}=\left\{\left(\beta^{a}, \beta^{b}, \gamma\right) \in \mathbb{R}^{+l} \times \mathbb{R}^{+l} \times \mathbb{R}: a_{0}^{T}\left(\beta^{a}, \beta^{b}, \gamma\right) \leq\left\|a_{0}\right\|\|v\|+1,\left(\beta^{a}-\beta^{b}, \gamma\right) \in V\right\}
$$

is admissible, well bounded and well centered.

The vector $\beta^{a}$ (resp. $\beta^{b}$ ) represents the amount of assets bought (resp. sold). This gives a simple interpretation to Eq. 5.1. 


\subsection{Limiting the jump sizes or the realized volatility}

Limitations to the up and/or down jumps or to the realized volatility can simply be achieved by limiting the possible paths that $\left(X_{1}, \ldots, X_{m}\right)$ can take to those that respect these limitations.

\subsection{The single-maturity case}

Our results can be simplified when $m=1$. Let

$$
U=\left\{(\xi, \beta, \gamma) \in \mathbb{R}^{d} \times \mathbb{R}^{l} \times \mathbb{R}: \psi(\omega) \leq \xi \cdot X_{1}(\omega)+\beta \cdot \phi(\omega)+\gamma \text { for any } \omega \in \Omega\right\} .
$$

Using the same techniques as in the proof of theorem 3.2, the following can be shown.

Theorem 5.2. Assume the following:

1. The set $U$ is admissible and contains a known ball $B(u, r)$.

2. There is $\delta>0$ and a rational number $q$ such that, for

$$
v \in\left\{ \pm \delta e_{i}, 1 \leq i \leq d+l\right\}
$$

there is a probability $P$ with $E_{P}\left(X_{1}, \phi\right)=\left(X_{0}, \pi\right)+v$ and $q \leq E_{P}(\psi)$.

Let $a_{0}=\left(X_{0}, \pi, 1\right)$. Then

$$
\pi_{\text {sup }}=\inf _{(\xi, \beta, \gamma) \in U^{\prime}} \xi^{T} X_{0}+\beta^{T} \pi+\gamma
$$

where

$$
U^{\prime}=\left\{(\xi, \beta, \gamma) \in U: \xi^{T} X_{0}+\beta^{T} \pi+\gamma \leq a_{0}^{T} u+1\right\} .
$$

The set $U^{\prime}$ is admissible, well bounded and well centered.

Theorem 5.2 can for instance be used to calculate in polynomial time an optimal superreplicating price and corresponding strategy for the logarithmic function without the need for discretization. An alternative approach based on discretization and dynamic programming to optimally super-replicate the logarithmic function is given in (Davis, Obloj and Raval 2010).

\section{Replication of forward start options via semi-definite and lin- ear programming}

The condition that a polynomial of given degree is non-negative on a given interval has been shown to be equivalent (Bertsimas and Popescu 2002, Alizadeh, Eckstein, Noyan and Rudolf 2008) to a semidefinite constraint on the polynomial coefficients. The following proposition gives a simple proof of this result when the degree of the polynomial is 2 .

Proposition 6.1. The function $g(x)=u x^{2}+v x+w$ is nonnegative on the interval $[0, \infty)$ if and only if there exists $\delta \in \mathbb{R}$ such that

$$
\delta \leq v \text { and }\left[\begin{array}{cc}
2 u & \delta \\
\delta & 2 w
\end{array}\right] \geq 0
$$

The function $g$ is nonnegative on the interval $[\alpha, \beta]$ if and only if there exists $\delta \in \mathbb{R}$ such that $\delta \leq 4 g((\alpha+\beta) / 2)-g(\alpha)-g(\beta)$ and

$$
\left[\begin{array}{cc}
2 g(\alpha) & \delta \\
\delta & 2 g(\beta)
\end{array}\right] \geq 0 .
$$


Proof. We prove the first part. Suppose that $g$ is nonnegative on the interval $[0, \infty)$. Then $u \geq 0$ and $w \geq 0$. If $u>0$ then, since $g(\sqrt{w / u}) \geq 0$, Eq. 6.1 holds when $\delta=-2 \sqrt{u w}$. If $u=0$ then Eq. 6.1 holds when $\delta=0$. Conversely, if there is $\delta \in \mathbb{R}$ such that Eq. 6.1 holds, then the relation

$$
u x^{2}+\delta x+w=\frac{1}{2}\left[\begin{array}{ll}
x & 1
\end{array}\right]\left[\begin{array}{cc}
2 u & \delta \\
\delta & 2 w
\end{array}\right]\left[\begin{array}{l}
x \\
1
\end{array}\right]
$$

shows that $g$ is nonnegative on the interval $[0, \infty)$.

The second part of the proposition follows from the first by observing that $g$ is nonnegative on the interval $[\alpha, \beta]$ if and only if $g(\alpha+(\beta-\alpha) z /(1+z)) \geq 0$ for $z \geq 0$. This is equivalent to $u^{\prime} z^{2}+v^{\prime} z+w \geq 0$ for $z \geq 0$, where $u^{\prime}=g(\beta), v^{\prime}=2 \alpha \beta u+(\alpha+\beta) v+2 w$ and $w^{\prime}=g(\alpha)$.

Theorem 6.1. Optimal super-replicating (resp. sub-replicating) prices and strategies for the forward start option can be calculated using semi-definite (resp. linear) programming.

Proof. We set $K_{i, 0}=0$ for $1 \leq i \leq 2$. For simplicity of notation, assume the forward start option $\psi$ is an at the money call. Extending the proof to the general case is straightforward. We first show how to calculate an optimal super-replicating portfolio. Consider a portfolio long of $\gamma$ bonds that pay 1 at time-step 2 and of $b_{i, j}$ calls of strike $K_{i, j}$ and maturity $T_{i}$, with $1 \leq i \leq 2$ and $1 \leq j \leq l_{i}$. Denote by $b_{i}\left(\right.$ resp. $\left.c_{i}\right)$ the vector $\left(b_{i, j}\right)\left(\right.$ resp. $\left.\left(c_{i, j}\right)\right), 1 \leq j \leq l_{i}$. The portfolio costs $\gamma+b_{1}^{T} c_{1}+b_{2}^{T} c_{2}$. It can be combined with a dynamic position in the stock to super-replicate $\psi$ if and only if there is a constant $b_{0}$ and a function $\xi_{2}$ of $S_{1}$ such that, for $x_{1} \geq 0$ and $x_{2} \geq 0$,

$$
\Psi^{*}\left(x_{1}, x_{2}\right) \leq \gamma+b_{0}\left(x_{1}-x_{0}\right)+\xi_{2}\left(x_{1}\right)\left(x_{2}-x_{1}\right),
$$

where $\Psi^{*}$ is given by Eq. 4.1 and $f_{1}$ and $f_{2}$ are defined as in section 4 . Let $b=\left(b_{0}, b_{1}, b_{2}\right)$ and

$$
H_{\gamma, b}\left(x_{1}, x_{2}\right)=\Psi^{*}\left(x_{1}, x_{2}\right)-\gamma-b_{0}\left(x_{1}-x_{0}\right) .
$$

Eq. 6.3 is equivalent to the condition that, for $x_{1} \geq 0$, the concave envelope of the function $H_{\gamma, b}\left(x_{1},.\right)$ at $x_{1}$ is at most 0 . Given $x_{1} \geq 0$, the function $H_{\gamma, b}\left(x_{1},.\right)$ is continuous and affine on any sub-interval of $\mathbb{R}^{+}-\left\{x_{1}\right\}$ containing no strike $K_{2, j}$. The concave envelope of $H_{\gamma, b}\left(x_{1},.\right)$ can hence be determined solely from its values at $x_{1}$ and $K_{2, j}$ and from its asymptotic slope $b_{2}^{T} \mathbf{1}-1$. Thus Eq. 6.3 is equivalent to

$$
H_{\gamma, b}\left(x_{1}, x_{1}\right) \leq 0
$$

for $x_{1} \geq 0$,

$$
H_{\gamma, b}\left(x_{1}, K_{2, j}\right)+\left(b_{2}^{T} \mathbf{1}-1\right)\left(K_{2, j}-x_{1}\right) \leq 0
$$

for $0 \leq j \leq l_{2}$ and $K_{2, j}<x_{1}$, and

$$
g_{\gamma, b, j, j^{\prime}}\left(x_{1}\right) \geq 0
$$

for $0 \leq j<j^{\prime} \leq l_{2}$ and $K_{2, j} \leq x_{1}<K_{2, j^{\prime}}$, where

$$
g_{\gamma, b, j, j^{\prime}}\left(x_{1}\right)=\left(x_{1}-K_{2, j^{\prime}}\right) H_{\gamma, b}\left(x_{1}, K_{2, j}\right)+\left(K_{2, j}-x_{1}\right) H_{\gamma, b}\left(x_{1}, K_{2, j^{\prime}}\right) .
$$

Equivalently,

$$
\begin{gathered}
b^{T} \mathbf{1} \geq 0, \\
H_{\gamma, b}\left(K_{i, j}, K_{i, j}\right) \leq 0
\end{gathered}
$$

for $1 \leq i \leq 2,0 \leq j \leq l_{i}$

$$
H_{\gamma, b}\left(K_{1, j}, K_{2, j^{\prime}}\right)+\left(b_{2}^{T} \mathbf{1}-1\right)\left(K_{2, j^{\prime}}-K_{1, j}\right) \leq 0
$$

for $1 \leq j \leq l_{1}, 0 \leq j^{\prime} \leq l_{2}$ with $K_{2, j^{\prime}}<K_{1, j}$, and

$$
g_{\gamma, b, j, j^{\prime}}\left(x_{1}\right) \geq 0
$$


for $0 \leq j<j^{\prime} \leq l_{2},\left[a_{h}, a_{h}^{\prime}\right] \in I_{j, j^{\prime}}$ and $x_{1} \in\left[a_{h}, a_{h}^{\prime}\right]$, where $I_{j, j^{\prime}}$ is the set of non-empty intervals of the form $\left[K_{2, j}, K_{2, j^{\prime}}\right] \cap\left[K_{1, j^{\prime \prime}}, K_{1, j^{\prime \prime}+1}\right]$. Observe that $g_{\gamma, b, j, j^{\prime}}$ is a quadratic function of $x_{1}$ on each element of $I_{j, j^{\prime}}$. By proposition 6.1, Eq. 6.7 is equivalent to the existence of $\delta_{j, j^{\prime}, h} \in \mathbb{R}$ such that

$$
\delta_{j, j^{\prime}, h} \leq 4 g_{\gamma, b, j, j^{\prime}}\left(\left(a_{h}+a_{h}^{\prime}\right) / 2\right)-g_{\gamma, b, j, j^{\prime}}\left(a_{h}\right)-g_{\gamma, b, j, j^{\prime}}\left(a_{h}^{\prime}\right)
$$

and

$$
\left[\begin{array}{cc}
2 g_{\gamma, b, j, j^{\prime}}\left(a_{h}\right) & \delta_{j, j^{\prime}, h} \\
\delta_{j, j^{\prime}, h} & 2 g_{\gamma, b, j, j^{\prime}}\left(a_{h}^{\prime}\right)
\end{array}\right] \geq 0
$$

In conclusion, $\pi_{\text {sup }}=\min _{b, \gamma, \delta} \gamma+b_{1}^{T} c_{1}+b_{2}^{T} c_{2}$, subject to $6.4,6.5,6.6,6.8$ and 6.9. Since $g_{\gamma, b, j, j^{\prime}}\left(x_{1}\right)$ is a linear functions of $(\gamma, b)$ for fixed $x_{1}$, this is a semidefinite program.

We can similarly calculate an optimal sub-replicating strategy by replacing $\psi$ with $-\psi$ and using remark 3.3. The corresponding function $g_{\gamma, b, j, j^{\prime}}$ is concave in this case, and so Eq. 6.7 is now equivalent to the condition that $g_{\gamma, b, j, j^{\prime}}$ is non-negative on the set $\left[K_{2, j}, K_{2, j^{\prime}}\right] \cap\left\{K_{1, j^{\prime \prime}}: 0 \leq\right.$ $\left.j^{\prime \prime} \leq l_{1}\right\}$. The corresponding optimization problem can thus be solved using linear programming.

\section{Conclusion}

We have shown that optimal super-replicating and sub-replicating prices and strategies can be calculated in polynomial time for a wide variety of exotic financial derivatives in terms of liquid financial derivatives in a multi-period setting. Our approach is based on convex programming and recursive calculations of concave envelopes. We have implemented our method using an analytic center cutting plane algorithm and an optimized convex hull algorithm. Numerical calculations with high accuracy of optimal super-replicating and sub-replicating prices in terms of call options were given for forward start options and variance and volatility swaps. These prices are close to the Black-Scholes prices in some cases and differ considerably from them in other cases. Our method can take into account interest rates and dividends, bid-ask spreads and limitations to the jumps or to the realized volatility of the underlying assets. It can be applied to multi-period financial derivatives on multiple assets but, in general, the corresponding running time is exponential in the number of assets. This is because, in general, the number of points needed to discretize the possible values of the assets vector is exponential in the number of assets. An alternative way to calculate optimal super-replicating and sub-replicating prices for forward-start options using semi-definite and linear programming was given.

\section{A Proof of theorem 2.1}

Denote by $d(y, C)$ the Euclidean distance from $y$ to $C$.

Definition A.1. The weak separation problem: given a vector $y \in \mathbb{Q}^{n}$ and a rational number $\epsilon>0$, either

1. assert that $d(y, C) \leq \epsilon$ or

2. find a vector $a \in \mathbb{Q}^{n}$ such that $\|a\| \geq 1$ and, for every $x \in C, a^{T} x \leq a^{T} y+\epsilon$.

We first show that $C$ admits a polynomial-time algorithm that solves the weak separation problem. Consider a ball $B(0, R)$ containing $C$. Let $y \in \mathbb{Q}^{n}$ and a rational number $\epsilon>0$. By hypothesis, there is a subset $D$ of $\mathbb{R}^{n} \times \mathbb{R}$ and a polynomial time algorithm that satisfy the conditions sated in definition 2.3. If the algorithm asserts that $y \in C$ then $d(y, C) \leq \epsilon$. Otherwise, the algorithm calculates $(a, b) \in D$ such that $a^{T} y \leq b+\epsilon$, and so it outputs a rational vector $\left(a^{*}, b^{*}\right)$ within distance $\epsilon$ from $(a, b)$. For $x \in C$, since $a^{T} x \geq b$, then $a^{T} y \leq a^{T} x+\epsilon$ and so, 
by the Cauchy-Schwarz inequality, $a^{* T} y \leq a^{* T} x+\epsilon+\epsilon R+\epsilon\|y\|$. Hence $C$ admits a polynomialtime weak separation algorithm. By (Grötschel, Lovász and Schrijver 1981, Theorem 2.4), this implies that the weak optimization problem can be solved for $C$ in polynomial time. In other words, there is a polynomial-time algorithm that, given a vector $a_{0} \in \mathbb{Q}^{n}$ and a rational number $\epsilon>0$, finds a vector $y \in \mathbb{Q}^{n}$ such that $d(y, C) \leq \epsilon$ and $a_{0}^{T} y \leq a_{0}^{T} x+\epsilon$ for every $x \in C$. Since condition 1 in definition 2.3 is slightly stronger than condition 1 in definition A.1, the proof of (Grötschel, Lovász and Schrijver 1981, Theorem 2.4) actually shows that $y \in C$.

On the other hand, the ellipsoid method described in (Grötschel, Lovász and Schrijver 1981, Theorem 2.4) calculates a sequence of vectors $\left(a_{k}, b_{k}\right)$ that belong to $D, 1 \leq k \leq l$, such that $a_{0}^{T} y \leq a_{0}^{T} x+\epsilon$ for any $x \in K$, where

$$
K=\left\{x \in \mathbb{R}^{n}: a_{k}^{T} x \geq b_{k} \text { for } 1 \leq k \leq l\right\} .
$$

Thus $a_{0}^{T} y \leq b_{0}+\epsilon$, where $b_{0}=\inf _{x \in K} a_{0}^{T} x$. By solving the dual linear program, we find non-negative weights $\lambda_{k}, 1 \leq k \leq l$, with

$$
\left(a_{0}, b_{0}\right)=\sum_{k=1}^{l} \lambda_{k}\left(a_{k}, b_{k}\right) .
$$

This concludes the proof.

\section{B Proof of theorem 3.2}

We first show the following.

Proposition B.1. Assume that $C$ contains a ball B(u,r) and is given by Eq. 2.1, where $D$ is a subset of $\mathbb{R}^{n} \times \mathbb{R}$. Let $a_{1} \in \mathbb{R}^{n-1}$ and $a_{0}=\left(a_{1}, 1\right)$. Assume further that, for some $\delta>0$ and some real number $q$, the vectors $\left(a_{0} \pm \delta e_{i}, q\right)$ belong to $D$ for $1 \leq i \leq n-1$. Then the set

$$
C^{\prime}=\left\{x \in C: a_{0}^{T} x \leq a_{0}^{T} u+1\right\}
$$

is well centered and well bounded.

Proof. Let $r^{\prime}$ be a positive rational number less than $\min \left(r,\left\|a_{0}\right\|^{-1}\right)$. Since $C^{\prime}$ contains $B\left(u, r^{\prime}\right)$, it is well centered. On the other hand, for $x \in C^{\prime}$ and $1 \leq i \leq n-1$,

$$
\begin{aligned}
q & \leq\left(a_{0} \pm \delta e_{i}\right)^{T} x \\
& \leq a_{0}^{T} u+1 \pm \delta x_{i},
\end{aligned}
$$

and so $\left|x_{i}\right| \leq \alpha$, where

$$
\alpha=\left(a_{0}^{T} u+1-q\right) / \delta .
$$

Thus,

$$
x_{n} \leq a_{0}^{T} u+1+\alpha\left\|a_{1}\right\|_{1} .
$$

Furthermore, since $\left(a_{0}, q\right)$ belongs to $D$,

$$
q-\alpha\left\|a_{1}\right\|_{1} \leq x_{n}
$$

Thus $C^{\prime}$ is well bounded. 
We now prove theorem 3.2. Proposition 2.1 shows that $V^{\prime}$ is admissible. We can apply Proposition B.1 with $a_{1}=\pi$ by noticing that $V$ is still equal to the right-hand side of Eq. 2.1 if we replace $D$ by

$$
D^{\prime}=\left\{\left(\left(E_{P}(\phi), 1\right), q^{\prime}\right): P \in \mathbb{P}, q^{\prime} \leq E_{P}(\psi)\right\} .
$$

This implies that $V^{\prime}$ is well bounded and well centered.

\section{Proof of proposition 3.1}

Since the market is arbitrage-free, it can be shown using classical techniques in multiperiod markets with a finite state-space (Pliska 2005, Section 3.4) that any underlying single-period market is arbitrage-free, and thus admits a risk-neutral probability. This implies that $\mathbb{P}(\theta)$ is non-empty and that $x_{i} \in \hat{D(\theta)}$. Let $g=\sum_{j=1}^{m} \xi_{j}^{T}\left(X_{j}-X_{j-1}\right)$ be a gains function such that $\psi$ is upper-bounded by $c(\psi)+\epsilon+g$. We show by backward induction that

$$
\psi_{i} \leq c(\psi)+\epsilon+\sum_{j=1}^{i} \xi_{j}^{T}\left(X_{j}-X_{j-1}\right) .
$$

Eq. C. 1 clearly holds when $i=m$. If it holds for $i+1$ then, for $\theta=\left(x_{1}, \ldots, x_{i}\right) \in D_{i}$ and $x \in D(\theta)$,

$$
\psi_{i+1}^{*}(\theta, x) \leq c(\psi)+\epsilon+\xi_{i+1}^{T}\left(x-x_{i}\right)+\sum_{j=1}^{i} \xi_{j}^{T}\left(x_{j}-x_{j-1}\right) .
$$

Since the right-hand side of C.2 is a linear function of $x$, we conclude that it is an upper bound on $\overline{\psi_{i+1}^{*}(\theta, .)}(x)$ for $x \in \hat{D(\theta)}$. Hence $\psi_{i}$ is well defined and Eq. C.1 holds for $i$. Thus $\psi_{0} \leq c(\psi)$.

Conversely, it follows from the definition of $\psi_{i}^{*}$ and Eq. 3.5 that there is a random variable $\xi_{i+1}$ which is a function of $X_{0}, \ldots, X_{i}$ such that Eq. 3.8 holds. Hence there is a gains function $g$ such that $\psi \leq m \epsilon+g+\psi_{0}$. We conclude that $\psi_{0}=c(\psi)$. Eq 3.7 follows immediately from Eq. 3.4.

\section{Proof of proposition 3.2}

For $\left(x_{1}, \ldots, x_{m}\right) \in D_{m}$, choose a state $\omega \in \Omega$ such that $X_{j}(\omega)=x_{j}$ for $1 \leq j \leq i$ and set

$$
P(\{\omega\})=\prod_{i=1}^{m} \mathcal{P}_{\left(x_{1}, \ldots, x_{i-1}\right)}\left(\left\{x_{i}\right\}\right) .
$$

The reader can verify that $P$ is a probability. The relation $E_{P}(\phi)=\bar{\phi}_{0}(\emptyset)$ then holds if $\phi^{*}$ is the indicator function of a path $\theta \in D_{m}$ and so, by linearity of expectations, it holds for any function $\phi^{*}$. Consider now the case where $\phi=\sum_{j=1}^{m} \xi_{j} .\left(X_{j}-X_{j-1}\right)$ is a gains function. Using backward induction and Eq. 3.9, it can be shown that $\bar{\phi}_{i}(\theta)=\sum_{j=1}^{i} \xi_{j} \cdot\left(x_{j}-x_{j-1}\right)$ for $\theta=\left(x_{1}, \ldots, x_{i}\right) \in D_{i}$, and so $E_{P}(\phi)=0$. Thus $P$ is risk-neutral. Furthermore, it can be shown by backward induction that $\psi_{i}^{*} \leq \bar{\psi}_{i}+(m-i) \epsilon$, where $\bar{\psi}_{i}$ is the sequence obtained by replacing $\phi$ with $\psi$ in Eq. 3.9, and so $\psi_{0} \leq E_{P}(\psi)+m \epsilon$. 


\section{References}

Albrecher, H., Mayer, P. A. and Schoutens, W. (2008). General lower bounds for arithmetic asian option prices., Applied Mathematical Finance 15(2): 123 - 149.

Alizadeh, F., Eckstein, J., Noyan, N. and Rudolf, G. (2008). Arrival Rate Approximation by Nonnegative Cubic Splines, Operations Research 56(1): 140-156.

Andrew, A. M. (1979). Another efficient algorithm for convex hulls in two dimensions, Inf. Process. Lett. 9(5): 216-219.

Bertsimas, D. and Popescu, I. (2002). On the relation between option and stock prices: a convex optimization approach, Operations Research 50(2): 358-374.

Boyle, P. P. and Lin, X. S. (1997). Bounds on contingent claims based on several assets, Journal of Financial Economics 46(3): 383-400.

Britten-Jones, M. and A. Neuberger, A. (2000). Option prices, implied price processes, and stochastic volatility, Journal of Finance 55(2): 839-66.

Brown, H., Hobson, D. and Rogers, L. C. G. (2001). Robust hedging of barrier options, Math. Finance 11(3): 285-314.

Carassus, L., Gobet, E. and Temam, E. (2007). A class of financial products and models where super-replication prices are explicit, in J. Akahori, S. Ogawa and S. Watanabe (eds), Proc. Ritsumeikan Int. Symp. on Stoch. Proc. and Apps to Math. Fin., World Scientific, Kusatsu, Japan, pp. 67-84.

Committee on Banking Supervision, B. (2009). Revisions to the Basel II market risk framework, Technical report, Bank for International Settlements.

Davis, M. H. A. and Hobson, D. G. (2007). The range of traded option prices, Math. Finance 17(1): $1-14$.

Davis, M. H. A., Obloj, J. and Raval, V. (2010). Arbitrage Bounds for Prices of Weighted Variance Swaps, ArXiv e-prints .

Dupire, B. (1993). Model art, Risk pp. 118-120.

Gotoh, J.-y. and Konno, H. (2002). Bounding option prices by semidefinite programming: A cutting plane algorithm, Management Science 48(5): 665-678.

Grötschel, M., Lovász, L. and Schrijver, A. (1981). The ellipsoid method and its consequences in combinatorial optimization, Combinatorica 1(2): 169-197.

Grundy, B. D. (1991). Option prices and the underlying asset's return distribution, Journal of Finance 46(3): 1045 - 1069.

Hobson, D. G. (1998). Robust hedging of the lookback option, Finance and Stochastics 2(4): 329-347.

Hobson, D. and Klimmek, M. (2012). Model-independent hedging strategies for variance swaps, Finance and Stochastics 16: 611-649.

Hobson, D. and Neuberger, A. (2010). Robust bounds for forward start options, Math. Finance 22(1): $31-56$. 
Kahale, N. (2009). Model-independent lower bound on variance swaps. http://ssrn.com/abstract $=1493722$.

Kahale, N. (2010). Sparse calibrations of contingent claims, Math. Finance 20(1): 105-115.

Lo, A. W. (1987). Semi-parametric upper bounds for option prices and expected payoffs, Journal of Financial Economics 19(2): 373-387.

Neuberger, A. J. (1994). The log contract, Journal of Portfolio Management 20(2): 74-80.

Pliska, S. (2005). Introduction to Mathematical Finance, Blackwell, Malden, MA. 